\title{
The effects of tantalum addition on the microtexture and mechanical behaviour of tungsten for ITER applications
}

\author{
E. Tejado a, b, *, P.A. Carvalho ${ }^{\text {c, d }}$, A. Munoz ${ }^{\text {e }}$, M. Dias ${ }^{\text {c }}$, J.B. Correia ${ }^{\text {c, f }, ~ U . V . ~ M a r d o l c a r ~}{ }^{\text {c, g, }}$, \\ J.Y. Pastor ${ }^{\mathrm{a}}$ \\ a Departamento de Ciencia de Materiales-CIME, ETSI Caminos, Canales y Puertos, Universidad Politécnica de Madrid, Madrid, Spain \\ b Centro Nacional de Investigaciones Metalúrgicas (CSIC), Madrid, Spain \\ c Associação Euratom/IST, Instituto de Plasmas e Fusão Nuclear, Instituto Superior Técnico, Universidade Técnica de Lisboa, Av. Rovisco Pais, $1049-001$ \\ Lisboa, Portugal \\ d ICEMS, Departamento de Bioengenharia, Instituto Superior Técnico, Av. Rovisco Pais, 1049-001 Lisboa, Portugal \\ e Departamento de Física, Universidad Carlos III, Leganés, Spain \\ ${ }^{\mathrm{f}}$ LNEG, Laboratório Nacional de Energia e Geologia, Estrada do Paço do Lumiar, 1649-038 Lisboa, Portugal \\ ${ }^{g}$ Centro de Química Estrutural, Instituto Superior Técnico, Universidade de Lisboa, Av. Rovisco Pais, 1049-001 Lisboa, Portugal
}

\section{A R T I C L E I N F O}

\section{Article history:}

Received 3 March 2015

Received in revised form

19 October 2015

Accepted 22 October 2015

Available online 24 October 2015

\section{Keywords:}

Tungsten

Tantalum

Fracture toughness

Mechanical properties

High temperature

Nuclear applications

\begin{abstract}
A B S T R A C T
Tungsten (W) and its alloys are very promising materials for producing plasma-facing components (PFCs) in the fusion power reactors of the near future, even as a structural part in them. However, whereas the properties of pure tungsten are suitable for a PFC, its structural applications are still limited due to its low toughness, ductile to brittle transition temperature and recrystallization behaviour. Therefore, many efforts have been made to improve its performance by alloying tungsten with other elements.

Hence, in this investigation, the thermo-mechanical performance of two new tungsten-tantalum materials has been evaluated. Materials with $\mathrm{W}-5 \mathrm{wt} . \% \mathrm{Ta}$ and $\mathrm{W}-15 \mathrm{wt} . \% \mathrm{Ta}$ were processed by mechanical alloying (MA) and later consolidation by hot isostatic pressing (HIP), with distinct settings for each composition. Thus, it was possible to determine the relationship between the microstructure and the addition of Ta with the macroscopic mechanical properties. These were measured by means of hardness, flexural strength and fracture toughness, in the temperature range of 300-1473 K. The microstructure and the fracture surfaces features of the tested materials were analysed by Field Emission Scanning Electron Microscopy (FESEM).
\end{abstract}

(C) 2015 Elsevier B.V. All rights reserved.

\section{Introduction}

Nuclear fusion is the least developed of the alternatives to nonrenewable energy resources, although it has a high potential for future energy supply since its raw materials are abundant and widely available. However, one of the main obstacles faced for the development of a fusion plant is the designing of materials that will tolerate the harsh radiation environment combined with a strong mechanical stress [1]. Tungsten and tungsten alloys are some of the candidate armour materials for the plasma-facing components of the International Thermonuclear Experimental Reactor and its

\footnotetext{
Presented at the NuMat 2014 Conference, 27-30 October 2014, Clearwater, Florida, USA

* Corresponding author. Departamento de Ciencia de Materiales-CIME, ETSI Caminos, Canales y Puertos, Universidad Politécnica de Madrid, Madrid Spain.

E-mail address: elena.tejado@mater.upm.es (E. Tejado).
}

previous prototype DEMO. For the present reference design, tungsten has been selected as the armour for the divertor, the upper vertical target, the dome, the cassette liner, and for the lower baffle because of its unique resistance to ion and charge-exchange particle erosion in comparison with other materials [2]. For these applications, different tungsten grades (pure, dispersion strengthened and cast alloys) have been considered. However, the mechanical properties of commercially available tungsten are not yet adequate for structural purposes due to the intrinsic brittleness of tungsten at relatively low temperatures, as the ductile-brittle transition (DBTT) occurs in the vicinity of $0.15 \mathrm{~T}_{\mathrm{m}}$, thus limiting the operating temperatures of the reactor. On the other hand, the mechanical properties of $\mathrm{W}$ at high temperatures are quite poor, and it exhibits a strong degradation in air due to its linear oxidation.

The approaches towards reducing brittleness of $\mathrm{W}$ at low temperatures have not changed since 1975 , as reported by W.D. Klopp [3]. These are: i) improvement of purity, ii) maintenance of a fine- 